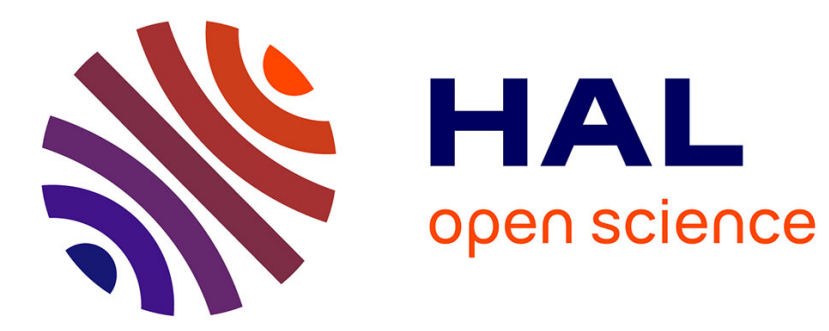

\title{
ASAP Top-k Query Processing in Unstructured P2P Systems
}

William Kokou Dedzoe, Philippe Lamarre, Reza Akbarinia, Patrick Valduriez

\section{To cite this version:}

William Kokou Dedzoe, Philippe Lamarre, Reza Akbarinia, Patrick Valduriez. ASAP Top-k Query Processing in Unstructured P2P Systems. IEEE Tenth International Conference on Peer-To-Peer Computing (P2P), Aug 2011, Kyoto, Netherlands. pp.1-10. lirmm-00607926

\section{HAL Id: lirmm-00607926 https://hal-lirmm.ccsd.cnrs.fr/lirmm-00607926}

Submitted on 11 Jul 2011

HAL is a multi-disciplinary open access archive for the deposit and dissemination of scientific research documents, whether they are published or not. The documents may come from teaching and research institutions in France or abroad, or from public or private research centers.
L'archive ouverte pluridisciplinaire HAL, est destinée au dépôt et à la diffusion de documents scientifiques de niveau recherche, publiés ou non, émanant des établissements d'enseignement et de recherche français ou étrangers, des laboratoires publics ou privés.

\section{(c)(1)}

Distributed under a Creative Commons Attribution| 4.0 International License 


\title{
ASAP Top- $k$ Query Processing in Unstructured P2P Systems
}

\author{
William Kokou Dedzoe*, Philippe Lamarre*, Reza Akbarinia*, Patrick Valduriez ${ }^{\dagger}$ \\ INRIA and LINA, University of Nantes, France* \\ INRIA and LIRMM, France ${ }^{\dagger}$ \\ emails: \{FirstName.LastName@univ-nantes.fr $\}^{*}$, Patrick.Valduriez@inria.fr ${ }^{\dagger}$
}

\begin{abstract}
Top- $k$ query processing techniques are useful in unstructured peer-to-peer (P2P) systems, to avoid overwhelming users with too many results. However, existing approaches suffer from long waiting times. This is because top- $k$ results are returned only when all queried peers have finished processing the query. As a result, query response time is dominated by the slowest queried peer. In this paper, we address this users' waiting time problem. For this, we revisit top- $k$ query processing in P2P systems by introducing two novel notions in addition to response time: the stabilization time and the cumulative quality gap. Using these notions, we formally define the as-soon-as-possible (ASAP) top- $k$ processing problem. Then, we propose a family of algorithms called ASAP to deal with this problem. We validated our solution through implementation and extensive experimentation. The results show that ASAP significantly outperforms baseline algorithms by returning final top- $k$ result to users in much better times.
\end{abstract}

\section{INTRODUCTION}

Unstructured Peer-to-Peer (P2P) networks have been widely used for sharing resources and content over the Internet [1], [2], [3]. In these systems, there is neither a centralized directory nor any control over the network topology or resource placement. Because of few topological constraints, they require little maintenance in highly dynamic environnements [4]. However, executing queries over unstructured P2P systems typically by flooding may incur high network traffic and produce lots of query results.

To reduce network traffic and avoid overwhelming users with high numbers of query results, complex query processing techniques based on top- $k$ answers have been proposed e.g. in [5]. With a top- $k$ query, the user specifies a number $k$ of the most relevant answers to be returned by the system. The quality (i.e. score of relevance) of the answers to the query is determined by user-specified scoring functions [6], [7]. Despite the fact that these top- $k$ query processing solutions reduce network traffic, they may significantly delay the answers to users. This is because top- $k$ results are returned to the user only when all queried peers have finished processing the query. Thus, query response time is dominated by the slowest queried peer, which makes users suffer from long waiting times. Therefore, these solutions are not suitable for emerging applications such as $\mathrm{P} 2 \mathrm{P}$ data sharing for online communities, which may have high numbers of autonomous data sources with various access performance. Most of the previous work on top- $k$ processing have focused on efficiently computing the exact or approximate result sets and reducing network traffic [8], [9], [10], [11], [5].
A naive solution to reduce users' waiting time is to have each peer return its top- $k$ results directly to the query originator as soon as it is done executing the query. However, this significantly increases network traffic and may cause a bottleneck at the query originator when returning high numbers of results. In this paper, we aim at reducing users' waiting time by returning high quality intermediate results, while avoiding high network traffic. The intermediate results are the results of peers which have already processed locally their query. Providing intermediate results to users is quite challenging because a naive solution may saturate users with results of low quality, and incur significant network traffic which in turn may increase query response time.

In this paper, our objective is to return high quality results to users as soon as possible. For this, we revisit top- $k$ query processing in $\mathrm{P} 2 \mathrm{P}$ systems by introducing two notions to complement response time: stabilization time and cumulative quality gap. The stabilization time is the time needed to obtain the final top- $k$ result set, which may be much lower than the response time (when it is sure that there is no other top- $k$ result). The quality gap of the top- $k$ intermediate result set is the quality that remains to be the final top- $k$ result set. The cumulative quality gap is the sum of the quality gaps of all top- $k$ intermediate result sets during query execution.

In summary, this paper makes the following contributions:

- We formally define as soon as possible top- $k$ query processing in large P2P systems based on both stabilization time and cumulative quality gap.

- We propose, a family of efficient algorithms called As Soon As Possible (ASAP). ASAP uses a threshold-based scheme that considers the score and rank of intermediate results to return quickly high quality results to users.

- We analytically evaluate ASAP's communication cost in terms of numbers of answer messages and volume of transferred data.

- We validated our solution through implementation and extensive experimentation. Our performance evaluation shows that ASAP significantly outperforms baseline algorithms by returning faster the final top- $k$ results. It also shows that ASAP achieves a good trade-off between the time to receive all the final top- $k$ results, the total number of intermediate results returned and the communication cost. Finally, the results demonstrate that in the presence of peers' failures, ASAP provides approximative top- $k$ results with good accuracy compared to baseline algorithms. 
The rest of this paper is organized as follows. In section II, we propose a model for unstructured $\mathrm{P} 2 \mathrm{P}$ systems and present basic definitions regarding top- $k$ queries in P2P systems. Section III formally defines the as-soon-as-possible top- $k$ query processing problem. In Section IV, we give an overview of ASAP top$k$ query processing. Section V presents ASAP approaches for bubbling up as soon as possible high quality results. In Section VI, we analytically evaluate the communication cost of ASAP. Section VII gives a performance evaluation of ASAP. In Section VIII, we discuss related work. Section IX concludes.

\section{SYSTEM MODEL}

In this section, we first present a general model of unstructured $\mathrm{P} 2 \mathrm{P}$ systems which is needed for describing our solution. Then, we provide a model and definitions for top- $k$ queries.

\section{A. Unstructured P2P Model}

We model an unstructured P2P network of $n$ peers as an undirected graph $G=(P, E)$, where $P=\left\{p_{0}, p_{1}, \cdots, p_{n-1}\right\}$ is the set of peers and $E$ the set of connections between the peers. For $p_{i}, p_{j} \in P,\left(p_{i}, p_{j}\right) \in E$ denotes that $p_{i}$ and $p_{j}$ are neighbours. We also denote by $N\left(p_{i}\right)$, the set of peers to which $p_{i}$ is directly connected, so $N\left(p_{i}\right)=\left\{p_{j} \mid\left(p_{i}, p_{j}\right) \in E\right\}$. The value $\left\|N\left(p_{i}\right)\right\|$ is called the degree of $p_{i}$. The average degree of peers in $G$ is called the average degree of $G$ and is denoted by $\varphi$. The $r$-neighborhood $N^{r}(p)(r \in \mathbb{N})$ of a peer $p \in P$ is defined as the set of peers which are at most $r$ hops away from peer $p$, so

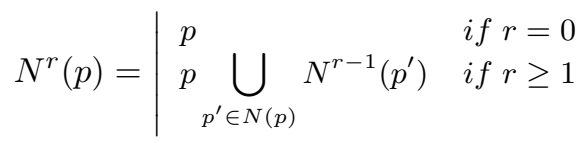

Each peer $p \in P$ holds and maintains a set $D(p)$ of data items such as images, documents or relational data (i.e. tuples). We denote by $D^{r}(p)(r \in \mathbb{N})$, the set of all data items which are in $N^{r}(p)$, so

$$
D^{r}(p)=\bigcup_{p^{\prime} \in N^{r}(p)} D\left(p^{\prime}\right)
$$

In our model, the query is forwarded from the query originator to its neighbours until the Time-To-Live value of the query decreases to 0 or the current peer has no peer to forward the query. So the query processing flow can be represented as a tree, which is called the query forwarding tree. When a peer $p_{0} \in P$ issues query $q$ to peers in its r-neighborhood, the results of these peers are bubbled up using query $q$ 's forwarding tree with root $p_{0}$ including all the peers belonging to $N^{r}\left(p_{0}\right)$. The set of children of a peer $p \in N^{r}\left(p_{0}\right)$ in query $q$ 's forwarding tree is denoted by $\psi(p, q)$.

\section{B. Top-k Queries}

We characterize each top- $k \quad q$ by a tuple $<q i d, c, t t l, k, f, p_{0}>$ such that qid is the query identifier, $c$ is the query itself (e.g. SQL query), $t t l \in \mathbb{N}$ (Time-To-Live) is the maximum hop distance set by the user, $k \in \mathbb{N}^{*}$ is the number of results requested by the user, $f: \mathcal{D} \times \mathcal{Q} \rightarrow[0,1]$ is a scoring function that denotes the score of relevance (i.e. the quality) of a given data item with respect to a given query and $p_{0} \in P$ the originator of query $q$, where $\mathcal{D}$ is the set of data items and $\mathcal{Q}$ the set of queries.

A top- $k$ result set of a given query $q$ is the $k$ top results among data items owned by all peers that receive $q$. Formally we define this as follows.

Definition 1: Top- $k$ Result Set. Given a top- $k$ query $q$, let $D^{\prime}=D^{q \cdot t t l}\left(q \cdot p_{0}\right)$. The top- $k$ result set of $q$, denoted by $\operatorname{Top}^{k}\left(D^{\prime}, q\right)$, is a sorted set on the score (in decreasing order) such that:

1) $T o p^{k}\left(D^{\prime}, q\right) \subseteq D^{\prime}$

2) If $\left\|D^{\prime}\right\|<q . k, \operatorname{Top}^{k}\left(D^{\prime}, q\right)=D^{\prime}$, otherwise $\left\|\operatorname{Top}^{k}\left(D^{\prime}, q\right)\right\|=q . k$;

3) $\forall d \in T o p^{k}\left(D^{\prime}, q\right), \forall d^{\prime} \in D^{\prime} \backslash T o p^{k}\left(D^{\prime}, q\right)$, $q . f(d, q . c) \geq q \cdot f\left(d^{\prime}, q . c\right)$

Definition 2: Result's Rank. Given a top- $k$ Result set $I$. We define the rank of result $d \in I$, denoted by $\operatorname{rank}(d, I)$, as the position of $d$ in the set $I$.

Note that the rank of a given top- $k$ item is in the interval $[1 ; k]$.

In large unstructured P2P systems, peers have different processing capabilities and store different volumes of data. In addition, peers are autonomous in allocating the resources to process a given query. Thus, some peers may process more quickly a given query than others. Intuitively, the top- $k$ intermediate result set for a given peer is the $k$ best results of both the results the peer received so far from its children and its local results (if any). Formally, we define this as follows.

Definition 3: Top- $k$ Intermediate Result Set. Given a top$k$ query $q$, and $p \in N^{q . t t l}\left(q \cdot p_{0}\right)$. Let $D_{1}$ be the result set of $q$ received so far by $p$ from peers in $\psi(p, q)$ and $D_{2}=D_{1} \cup D(p)$. The top- $k$ intermediate result set of $q$ at peer $p$, denoted by $I_{q}(p)$, is such that:

$I_{q}(p)=\mid \begin{array}{ll}\operatorname{Top}^{k}\left(D_{2}, q\right) & \text { if } p \text { has already processed } q \text { locally } \\ \operatorname{Top}^{k}\left(D_{1}, q\right) & \text { otherwise }\end{array}$

\section{Problem Definition}

Let us first give our assumptions regarding schema management and the unstructured $\mathrm{P} 2 \mathrm{P}$ architecture. We assume that peers are able to express queries over their own schema without relying on a centralized global schema as in data integration systems [12]. Several solutions have been proposed to support decentralized schema mapping. However, this issue is out of scope of this paper and we assumed it is provided using one of the existing techniques, e.g. [13], [12] and [14]. We also assume that all peers in the system are trusted and cooperative. In the following, we first give some definitions which are useful to define the problem we focus and formally state the problem.

\section{A. Foundations}

To process a top- $k$ query in $\mathrm{P} 2 \mathrm{P}$ systems, an ASAP top- $k$ algorithm provides intermediate results to users as soon as peers process the query locally. This allows users to progressively see 


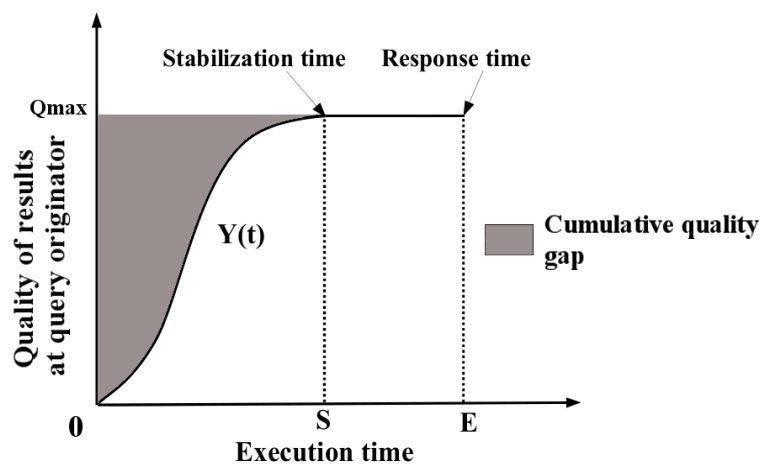

(a) Quality evolution.

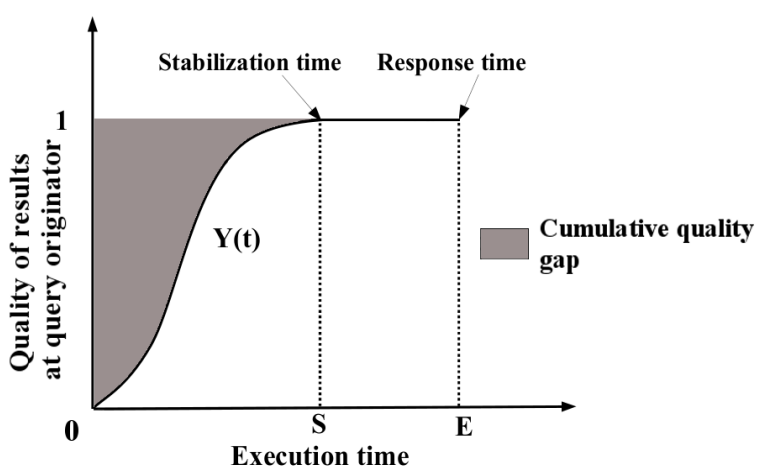

(b) Normalized Quality evolution.

Fig. 1. Quality of top- $k$ results at the query originator wrt. Execution time

the evolution of their query execution by receiving intermediate results for their queries. Note that at some point of query execution, the top- $k$ intermediate results received by a peer may not change any more, until the end of the query execution. We denote this point as the stabilization time.

Recall that the main goal of ASAP top- $k$ query processing is to return high-quality results to user as soon as possible. To reflect this, we introduce the quality evolution concept. Given a top- $k$ query $q$, we define the quality evolution $Y(t)$ of $q$ at time $t$ as the sum of scores of $q$ 's intermediate top- $k$ results at $t$ and at $q$ 's originator. Figure 1(a) shows the quality evolution of intermediate top- $k$ results obtained at the query originator during a given query execution. To be independent of the scoring values - which can be different from one query to another, we normalize the quality evolution of a query. With this in mind, we divide the quality evolution of a given query by the sum of scores of the final top- $k$ results of that query. Thus, the quality evolution values are in the interval $[0,1]$ and the quality of the top- $k$ final results are equal to 1 (see Figure 1(b)). Note that we do not use the proportion of the final top- $k$ results in intermediate top- $k$ results (i.e precision) to characterize ASAP algorithm because this metric does not express the fact of returning the high quality results as soon as possible to users.

The quality evolution of intermediate top- $k$ results at the query originator increases as peers answer a query. To reflect this, we introduce the cumulative quality gap, which is defined as the sum of the quality difference between intermediate top- $k$ result sets received until the stabilization time and the final top- $k$ result set (see Figure 1(b)). We formalize this in Definition 4.

Definition 4: Cumulative quality gap. Given a top- $k$ query $q$ and $Y(t)$, the quality evolution of $q$ at time $t$ at $q$ originator, let $S$ be the stabilization time of $q$. The cumulative quality gap of the query $q$, denoted by $C_{q g}$ is:

$$
C_{q g}=\int_{0}^{S}(1-Y(t)) \mathrm{d} t=S-\int_{0}^{S} Y(t) \mathrm{d} t
$$

\section{B. Problem Statement}

Formally, we define the ASAP top- $k$ query processing problem as follows. Given a top- $k$ query $q$, let $S$ be the stabilization time of $q$ and $C_{q g}$ be the cumulative quality gap of $q$. The problem is to minimize $C_{q g}$ and $S$ while avoiding high communication cost.

\section{AS AP TOP- $k$ Query Processing OVERVIEW}

ASAP query processing proceeds in two main phases. The first phase is the query forwarding and local execution of the query. The second phase is the bubbling up of the peers' results for the query along the query forwarding tree.

\section{A. Query Forwarding and Local Execution}

Query processing starts at the query originator, i.e. the peer at which a user issues a top- $k$ query $q$. The query originator performs some initialization. First, it sets $t t l$ which is either user-specified (or default). Second, it creates a unique identifier qid for $q$ which is useful to distinguish between new queries and those received before. Then, $q$ is included in a message that is broadcast by the query originator to its reachable neighbors. Algorithm 1 shows the pseudo-code of query forwarding. Each peer that receives the message including $q$ checks qid (see line 2, Algorithm 1). If it is the first time the peer has received $q$, it saves the query (i.e. saves the query in the list of seen queries and the address of the sender as its parent) and decreases the query $t t l$ by 1 (see lines 3-4, Algorithm 1). If the $t t l$ is greater than 0 , then the peer sends the query message to all neighbors except its parent (see lines 5-7, Algorithm 1). Then, it executes $q$ locally. If $q$ has been already received, then if the old $t t l$ is smaller than the new $t t l$, the peer proceeds as where $q$ is received for the first time but without executing $q$ locally (see lines 10-18, Algorithm 1), else the peer sends a duplicate message to the peer from which it has received $q$.

\section{B. Bubbling Up Results}

Recall that, when a peer submits a top- $k$ query $q$, the local results of the peers that received $q$ are bubbled up to the query originator using query $q$ 's forwarding tree. In ASAP, a peer's decision to send intermediate results is based on the improvement impact brought by its current top- $k$ intermediate result set over the top- $k$ intermediate result set it sent so far to its parent. This improvement impact can be computed in two ways: by using the score or rank of top- $k$ results in the result 


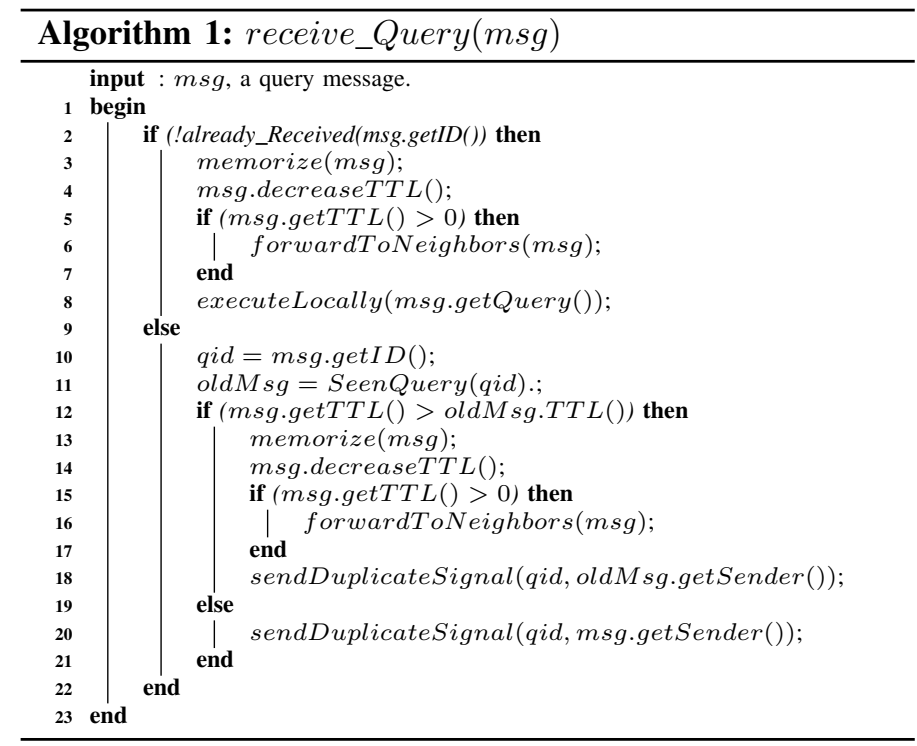

set. Therefore, we introduce two types of improvement impact: score-based improvement impact and rank-based improvement impact.

Intuitively, the score-based improvement impact at a given peer for a given top- $k$ query is the gain of score of that peer's current top- $k$ intermediate set compared to the top- $k$ intermediate set it sent so far.

Definition 5: Score-based improvement impact. Given a top- $k$ query $q$, and peer $p \in N^{q \cdot t t l}\left(q \cdot p_{0}\right)$, let $T_{c u r}$ be the current top- $k$ intermediate set of $q$ at $p$ and $T_{\text {old }}$ be the top- $k$ intermediate set of $q$ sent so far by $p$. The score-based improvement impact of $q$ at peer $p$, denoted by $I S \operatorname{Score}\left(T_{c u r}, T_{\text {old })}\right.$ is computed as

$$
\frac{\sum_{d \in T_{\text {cur }}} q \cdot f(d, q . c)-\sum_{d^{\prime} \in T_{\text {old }}} q \cdot f\left(d^{\prime}, q . c\right)}{k}
$$

Note that in Formula 2, we divide by $k$ instead of $\left\|T_{\text {cur }}-T_{\text {old }}\right\|$ because we do not want that $I S \operatorname{Score}\left(T_{\text {cur }}, T_{\text {old }}\right)$ be an average which would not be very sensitive to the values of scores. The score-based improvement impact values are in the interval $[0,1]$.

Intuitively, the rank-based improvement impact at a given peer for a given top- $k$ query is the loss of rank of results in the top- $k$ intermediate result set sent so far by that peer due to the arrival of new intermediate results.

Definition 6: Rank-based improvement impact. Given a top- $k$ query $q$ and peer $p \in N^{q . t t l}\left(q . p_{0}\right)$, let $T_{c u r}$ be the current top- $k$ intermediate result set of $q$ at $p$ and $T_{\text {old }}$ be the top- $k$ intermediate result set of $q$ sent so far by $p$. The rank-based improvement impact of $q$ at peer $p$, denoted by $\operatorname{IRank}\left(T_{\text {cur }}, T_{\text {old }}\right)$ is computed as

$$
\frac{\sum_{d \in T_{\text {cur } \backslash T_{\text {old }}}}^{\operatorname{Rank}\left(T_{\text {cur }}, T_{\text {old }}\right)}\left(k-\operatorname{rank}\left(d, T_{\text {cur }}\right)+1\right)}{\frac{k *(k+1)}{2}}
$$

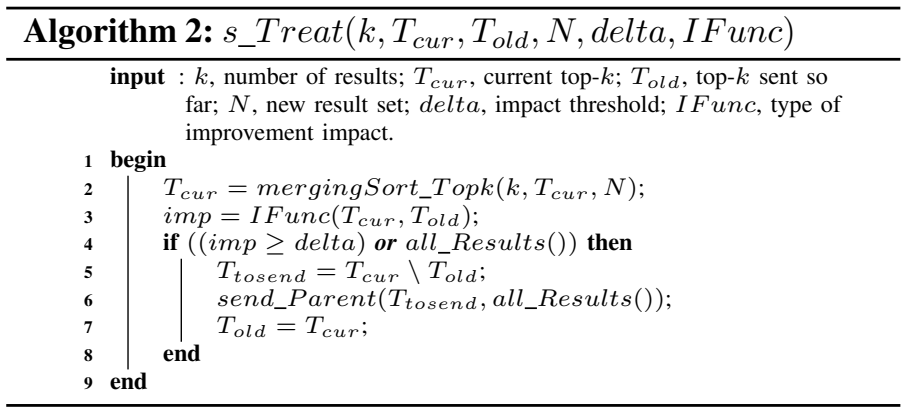

Note that in Formula 3, we divide by $\frac{k *(k+1)}{2}$ which is the sum of ranks of a set containing $k$ items. The rank-based improvement impact values are in the interval $[0,1]$.

Notice also that, in order to minimize network traffic, ASAP does not bubble up the results (which could be large), but only their scores and addresses. A score-list is simply a list of $k$ couples $(a d, s)$, such that $a d$ is the address of the peer owning the data item and $s$ its score.

\section{ASAP Threshold-BASED APPROACHES FOR BUBBLING UP RESULTS}

In this section, we present ASAP static and dynamic threshold-based approaches which use score and rank of intermediate results for bubbling up as-soon-as-possible high quality results.

\section{A. Static Approaches}

In these approaches, the minimum value that must reach the improvement impact before a peer sends newly received intermediate results to its parent is initially set by the application and it is the same for all peers in the system. Note also that this threshold does not change during the execution of the query. Using both types of improvement impact introduced in the previous section, we have two types of static threshold-based approaches. The first approach uses the score-based improvement impact and the second one the rank-based improvement impact.

A generic algorithm for our static threshold-based approaches is given in Algorithm 2. In these approaches, each peer maintains for each query a set $T_{\text {old }}$ of top- $k$ intermediate results sent so far to its parent and a set $T_{\text {cur }}$ of current top- $k$ intermediate results. When a peer receives a new result set $N$ from its children (or its own result set after local processing of a query), it first updates the set $T_{\text {cur }}$ with results in $N$ (see line 2, Algorithm 2). Then, it computes the improvement impact $i m p$ of $T_{\text {cur }}$ compared to $T_{\text {old }}$ (line 3 , Algorithm 2). If $i m p$ is greater than or equal to the defined threshold delta or if there are no more children' results to wait for, the peer sends the set $T_{\text {tosend }}=T_{\text {cur }} \backslash T_{\text {old }}$ to its parent and subsequently sets $T_{\text {curr }}$ to $T_{\text {old }}$ (see lines 4-7, Algorithm 2).

\section{B. Dynamic Approaches}

Although the static threshold-based approaches are interesting to provide results quickly to user, they may be blocking if results having higher scores are bubbled up before those of lower score. In other words, sending higher score's results will induce 
a decrease of improvement impact of the following results. This is because the improvement impact considered the top- $k$ intermediate results sent so far by the peer. Thus, results of low scores even if they are in the final top- $k$ results may be returned at the end of the query execution. To deal with this problem, an interesting way would be to have a dynamic threshold, i.e. a threshold that decreases as the query execution progresses. However, this would require finding the right parameter on which the threshold depends. We have identified two possible solutions for the dynamic threshold. The first one is to use an estimation of the query execution time. However, estimating the query execution time in large $\mathrm{P} 2 \mathrm{P}$ system is very difficult because it depends on network dynamics, such as connectivity, density, medium access contention, etc., and the slowest queried peer. The second, more practical, solution is to use for each peer the proportion of peers in its sub-tree including itself (i.e. all its descendants and itself) which have already processed the query to decrease the threshold.

1) Peer's Local Result Set Coverage:

Definition 7: Peer's local result set coverage. Given a top$k$ query, and $p \in N^{q . t t l}\left(q \cdot p_{0}\right)$, let $\mathcal{A}$ be the set of peers in the sub-tree whose root is $p$ in the query $q$ 's forwarding tree. Let $\mathcal{E}$ be the set of peers in $\mathcal{A}$ which have already processed $q$ locally. The local result set coverage of peer $p$ for $q$, denoted by $\operatorname{Cov}(\mathcal{E}, \mathcal{A})$, is computed using the following equation:

$$
\operatorname{Cov}(\mathcal{E}, \mathcal{A})=\frac{\|\mathcal{E}\|}{\|\mathcal{A}\|}
$$

Peer's local result set coverage values are in the interval $[0,1]$.

Note that is very difficult to have the exact value of a peer's local result set coverage without inducing an additional number of messages in the network. This is because each peer must send a message to its parent each time its local coverage result set value changes. Thus, when a peer at hop $m$ from query originator updates its local result coverage, $m$ messages will be sent over the network. To deal with this problem, an interesting solution is to have an estimation of this value instead of the exact value.

The estimation of peer's local result set coverage can be done using two different strategies: optimistic and pessimistic. In the optimistic strategy, each peer computes the initial value of its local result set coverage based only on its children nodes. This value is then updated progressively as the peers in its sub-tree bubble up their results. Indeed, each peer includes in each response message sent to its parent the number of peers in its sub-tree (including itself) which have already processed the query locally and the total number of peers in its sub-tree including itself. This couple of values is used in turn by its parent to estimate its local result set coverage. Contrary to the optimistic strategy, in the pessimistic strategy, the local result set coverage estimation is computed at the beginning by each peer based on the Time-To-Live received with the query and the average degree of peers in the system. As in the case of the optimistic strategy, this value is updated progressively as the peers in its sub-tree bubble up their results.

In our dynamic threshold-based approaches, we estimate a peer's local result set coverage using the pessimistic strategy because the estimation value is more stable than with the optimistic strategy. Now, let us give more details about how a peer's local result set coverage pessimistic estimation strategy is done.

2) Peer's Local Result set Coverage Pessimistic Estimation: In order to estimate its local result set coverage, each peer $p_{i}$ maintains for each top- $k$ query $q$ and for each child $p_{j}$ a set $\mathcal{C}_{1}$ of couples $\left(p_{j}, a\right)$ where $a \in \mathbb{N}$ is the number of peers in the sub-tree of peer $p_{j}$ including $p_{j}$ itself. $p_{i}$ maintains also a set $\mathcal{C}_{2}$ of couples $\left(p_{j}, e\right)$ where $e \in \mathbb{N}$ is the total number of peers in the sub-tree of peer $p_{j}$ including $p_{j}$ itself which have already processed locally $q$. Now let $t t l^{\prime}$ be the time-to-live with which $p_{i}$ received query $q$ and $\varphi$ be the average degree of peers in the system. At the beginning of query processing, for all children of $p_{i}, e=0$ and $a=\sum_{u=0}^{t t l^{\prime}-2} \varphi^{u}$. During query processing, when a child $p_{j}$ in $\psi\left(p_{i}, q\right)$ wants to send results to $p_{i}$, it inserts in the answer message its couple of values $(e, a)$. Once $p_{i}$ receives this message, it unpacks the message, gets these values (i.e. $e$ and a) and updates the sets $\mathcal{C}_{1}$ and $\mathcal{C}_{2}$. The local result set coverage of peer $p_{i}$ for the query $q$ is then estimated using Formula 4 .

$$
\widetilde{\operatorname{Cov}}\left(\mathcal{C}_{1}, \mathcal{C}_{2}\right)=\frac{\sum_{\left(p_{j}, e\right) \in \mathcal{C}_{1}} e}{\sum_{\left(p_{j}, a\right) \in \mathcal{C}_{2}} a}
$$

Note that peer's local result set coverage estimation values are in the interval $[0,1]$.

3) Dynamic Threshold Function: In the dynamic threshold approaches, the improvement impact threshold used by a peer at a given time $t$ of the query execution depends on its local result set coverage at that time. This improvement impact threshold decreases as the local result set coverage increases. A dynamic threshold function is a function that allows peers to set their improvement impact threshold for a given local result set coverage. Now let us define formally what the dynamic threshold function means in Definition 8.

Definition 8: Dynamic Threshold Function. Given a top- $k$ query $q$ and $p \in N^{q . t t l}\left(q . p_{0}\right)$, the improvement impact threshold used by $p$ during $q$ 's execution, is a monotonically decreasing function $H$ such that:

$$
H: \mid \begin{array}{lll}
{[0,1]} & \rightarrow & {[0,1]} \\
x & \mapsto & -\alpha * x+\alpha
\end{array}
$$

with $\alpha \in[0,1[$. Notice that $x$ is a peer's result set coverage at given time and $\alpha$ the initial improvement impact threshold (i.e. $H(0)=\alpha)$.

4) Reducing Communication Cost: Using a rank-based improvement impact has the drawback of not minimizing as much as possible network traffic. This is because the rank-based improvement impact value is equal to 1 (the maximum value it can reach) when a peer receives the first result set containing $k$ results (from one of its children or after local processing of a query). Thus, each peer always sends a message over the network when it receives the first result set containing $k$ results. To deal with this problem and thus reduce communication cost, 

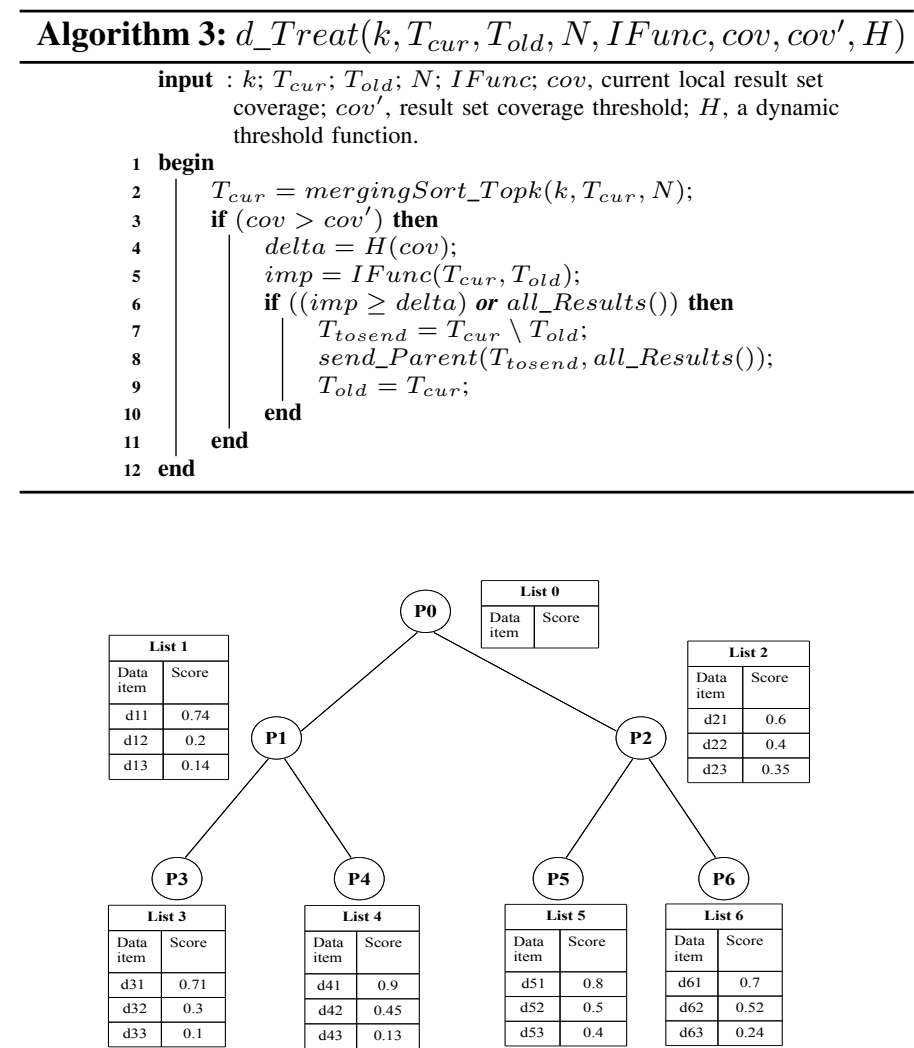

Fig. 2. Query forwarding tree of an example of unstructured P2P system

we use peers' result sets coverage to prevent them to send a message when they receive their first result set. Therefore, the idea is to allow peers to start sending a message if and only if their local result sets coverage reaches a predefined threshold. With this result set coverage threshold, peers send intermediate results based on the improvement impact threshold obtained from the dynamic threshold function $H$ define above.

5) Dynamic Threshold Approaches Algorithms: our dynamic threshold approaches algorithms are based on the same principles as the static threshold ones. A generic algorithm for our dynamic threshold-based approaches is given in Algorithm 3. When a peer receives a new result set $N$ from its children (or its own result set after local processing of a query), it first updates the set $T_{\text {cur }}$ of its current top- $k$ intermediate results with results in $N$ (see line 2, Algorithm 3). If its current result set coverage $c o v$ is greater than the defined threshold result set coverage $c o v^{\prime}$, then the peer computes the improvement threshold delta using the dynamic function $H$ and subsequently the improvement impact $i m p$ (see lines 3-5, Algorithm 3). If $i m p$ is greater than or equal to delta or if there are no more children' results to wait for, then the peer sends the set $T_{\text {tosend }}=T_{\text {cur }} \backslash T_{\text {old }}$ to its parent and subsequently sets $T_{\text {curr }}$ to $T_{\text {old }}$ (see lines 6-9, Algorithm 3). Recall that $T_{\text {cur }}$ is the set of the current top- $k$ intermediate results and $T_{\text {old }}$ is the top- $k$ intermediate results sent so far to its parent.

\section{Example}

To better illustrate ASAP top- $k$ processing, consider the query forwarding tree of a network graph consisting of seven peers $p_{0}, \cdots, p_{6}$ as shown in Figure 2. Let us assume that $p_{0}$ issues a top-3 query $q$ (i.e. $k=3$ ), and the end of local processing of $q$ at peers is in the following order $p_{0}, p_{4}, p_{1}, p_{5}, p_{3}, p_{6}$. Let list $_{0}, \cdots$, list $_{6}$ be respectively $p_{0} \cdots p_{6}$ top-3 lists after local processing of $q$. Due to space limitations, we only illustrate the ASAP static threshold-based approach which uses scorebased improvement impact and only on the portion $\left(p_{0}, p_{1}, p_{4}\right)$ of the query forwarding tree. We assume that the score-based improvement threshold is delta $=0.2$. The algorithm works as follows: after processing locally $q, p_{4}$ sends immediately list $_{4}$ to its parent $p_{1}$ (because $p_{4}$ has no children to wait for). Peer $p_{1}$, when receiving $p_{4}$ 's results, computes the score-based improvement impact of its current top-3 list compared with the top- $k$ list sent so far i.e. $\frac{(0.9+0.45+0.13)-(0)}{3}=0.493$. Since this value is greater than 0.2 , the predefined threshold, $p_{1}$ sends $l i s t_{4}$ to $p_{0}$. Once $p_{1}$ has completed the local processing of $q$, it computes the score-based improvement impact of its new current top-3 intermediate result set compared to list 4 (i.e. the top-3 it sent so far) as follows $\frac{(0.9+0.74+0.45)-(0.9+0.45+0.13)}{3}=0.203$. Because the value of improvement impact is greater than the predefined threshold, $p_{1}$ sends to $p_{0}$ the item in list $_{1}$ whose score is 0.74 .

\section{ASAP COST ANALYSIS}

In this section, we analytically evaluate the cost of ASAP in terms of number of answer messages and volume of transferred data (number of bytes) over the network to return final top- $k$ results to the user.

With ASAP, each peer sends a single answer message in the best case. Thus, if $n$ is the number of peers in the network, then the number of answer messages in the best case is equal to $n-1$. In the worst case, the number of answer messages sent by a peer depends on its depth in the query forwarding tree (i.e. the $t t l$ with which the peer receives the query). Let $P(i)$ be the number of peers at the hop $i$ in the query forwarding tree from initiator peer of a query $q$ with a Time-To-Live $t t l$. In the worst case the number of answer messages denoted by $n_{\text {asap }}^{\prime \prime}$ is:

$$
\begin{aligned}
n_{\text {asap }}^{\prime \prime}= & 0 * P(0)+1 * P(1)+2 * P(2)+\cdots \\
& +(t t l-2) * P(t t l-2)+t t l * P(t t l) \\
\leq & t t l * P(1)+t t l * P(2)+\cdots+t t l * P(t t l) \\
\leq & t t l *[P(1)+P(2)+\cdots+P(t t l)] \\
n_{\text {asap }}^{\prime \prime} \leq & t t l *(n-1) .
\end{aligned}
$$

To summarize, the number of answer messages sent by ASAP is such that:

$$
n-1 \leq n_{\text {asap }} \leq t t l *(n-1)
$$

Now let $k$ be the number of results request by the user and $z$ be the size in bytes of each element of a result set. In the best case the volume of transferred data over the network is equal to $k * z$. In the worst case, since the number of answer messages is $t t l *(n-1)$, the volume of transferred data over the network is equal to $k * z * t t l *(n-1)$. The volume of transferred data over the network in case of ASAP, denoted by $v_{a s a p}$, is:

$$
z * k \leq v_{\text {asap }} \leq k * z * t t l *(n-1)
$$

To summarize, the communication cost of ASAP in term of number of answer messages is $O(n)$ and the volume of transferred data over the network is $O(n * k)$. 


\section{Performance Evaluation}

In this section, we evaluate the performance of ASAP through simulation using the PeerSim simulator [15]. This section is organized as follows. First, we describe our simulation setup, the metrics used for performance evaluation. Then, we study the effect of the number of peers and the number of results on the performance of ASAP, and show how it scales up. Next, we study the effect of the number of replicas on the performance of ASAP. Finally, we investigate the effect of peers failures on the correctness of ASAP.

\section{A. Simulation Setup}

We implemented our simulation using the PeerSim simulator. PeerSim is an open source, Java based, P2P simulation framework aimed to develop and test any kind of P2P algorithm in a dynamic environment. It consists of configurable components and it has two types of engines: cycle-based and event-driven engine. PeerSim provides different modules that manage the overlay building process and the transport characteristics.

We conducted our experiments on a machine with a $2.4 \mathrm{GHz}$ Intel Pentium 4 processor and 2GB memory. The simulation parameters are shown in Table I. We use parameters values which are typical of $\mathrm{P} 2 \mathrm{P}$ systems [16]. The latency between any two peers is a normally distributed random number with mean of $200 \mathrm{~ms}$. Since users are usually interested in a small number of top results, we set $k=20$ as default value. In our experiments we vary the network size from 1000 to 10000 peers. In order to simulate high heterogeneity, we set peers' capacities in our experiments, in accordance to the results in [16]. This work measures the peers capacities in the Gnutella system. Based on these results, we generate around $10 \%$ of low-capable, $60 \%$ of medium-capable, and $30 \%$ of high-capable peers. The highcapable peers are 3 times more capable than medium-capable peers and still 7 times more capable than low-capable ones.

In the context of our simulations each peer in the P2P system has a table $\mathcal{R}($ data $)$ in which attribute data is a real value. The number of rows of $\mathcal{R}$ at each peer is a random number uniformly distributed over all peers greater than 1000 and less than 20000. In our experiments, we ensure that there is only one copy of each data item (i.e. tuple) in our system. We also ensure that there are not two different data items with the same score. In all our tests, we use the following simple query, denoted by $q_{l o a d}$ as workload:

SELECT val FROM R ORDER BY $F(\mathcal{R}$.data,val) STOP AFTER $k$

The scoring function we use is:

$$
F(x, y)=\frac{1}{1+|x-y|} \text { where }(x, y) \in \mathbb{R}^{2}
$$

In our simulation, we compare ASAP with Fully Distributed (FD) [5], a baseline approach for top- $k$ query processing in unstructured P2P systems which works as follows. Each peer that receives the query, executes it locally (i.e. selects the $k$ top scores), and waits for its children's results. After receiving all its children score-lists, the peer merges its $k$ local top data items with those received from its children and selects the $k$ top scores and sends the result to its parent.

\begin{tabular}{|l|l|}
\hline Parameters & Values \\
\hline Latency & $\begin{array}{l}\text { Normally distributed ran- } \\
\text { dom number, Mean }=200 \\
\text { ms, Variance }=100 \\
10,000 \text { peers }\end{array}$ \\
\hline Number of peers & 4 \\
\hline Average degree of peers & 9 \\
\hline$t t l$ & 20 \\
\hline$k$ & 1 \\
\hline Number of replicas & 1 \\
\hline
\end{tabular}

SiMULATION PARAMETERS.

In our experiments, to evaluate the performance of ASAP comparing to FD, we use the following metrics:

(i) Cumulative quality gap: As defined in Section III, is the sum of the quality difference between intermediate top- $k$ result sets received until the stabilization time and the final top- $k$ result set.

(ii) Stabilization time: We report on the stabilization time, the time of receiving all the final top- $k$ results.

(iii) Response time: We report on the response time, the time the query initiator has to wait until the top- $k$ query execution is finished.

(iv) Communication cost: We measure the communication cost in terms of number of answer messages and volume of data which must be transferred over the network in order to execute a top- $k$ query.

(v) Accuracy of results: We define the accuracy of results as follows. Given a top- $k$ query $q$, let $V$ be the set of the $k$ top results owned by the peers that received $q$, let $V^{\prime}$ be the set of top- $k$ results which are returned to the user as the response of the query $q$. We denote the accuracy of results by $a c_{q}$ and we define it as

$$
a c_{q}=\frac{\left\|V \cap V^{\prime}\right\|}{\|V\|}
$$

(iv) Total number of results: We measure the total number of results as the number of results received by the query originator during query execution.

In our experimentation, we perform 30 tests for each experiment by issuing $q_{\text {load }}$ at 20 different times and we report the average of their results. Due to space limitations, we only present the main results of ASAP's dynamic threshold-based approaches denoted by ASAP-Dscore and ASAP-Drank. ASAP-Dscore uses a score-based improvement impact and ASAP-Drank a rank-based improvement impact. ASAP's dynamic thresholdbased approaches have proved to be better than ASAP's static threshold-based approaches without being expensive in communication cost. In our all experiments in the case of ASAP-Dscore approach, we use $H(x)=-0.2 x+0.2$ as dynamic threshold function and 0 as peer's local result set coverage threshold. In the case Asap-Drank, we use $H(x)=-0.5 x+0.5$ as dynamic threshold function and 0.05 as peer's local result set coverage threshold.

\section{B. Performance Results}

1) Effect of number of peers: We study the effect of the number of peers on the performance of ASAP. For this, we ran experiments to study how cumulative quality gap, stabilization 


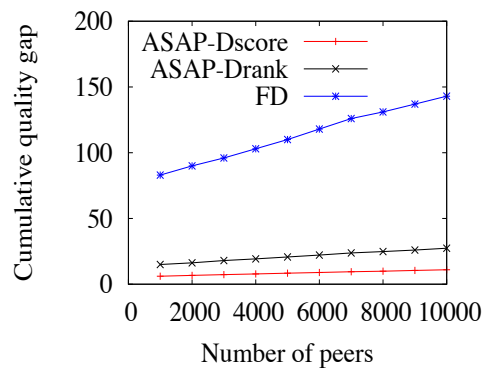

(a) Cumulative quality gap vs. Number of peers.

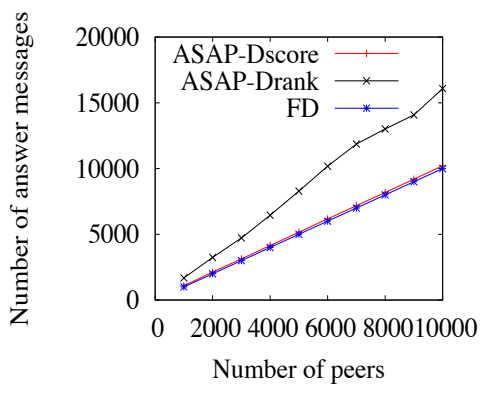

(d) Number of answer messages vs. Number of peers. (e) Volume of transferred data vs. Number of peers.

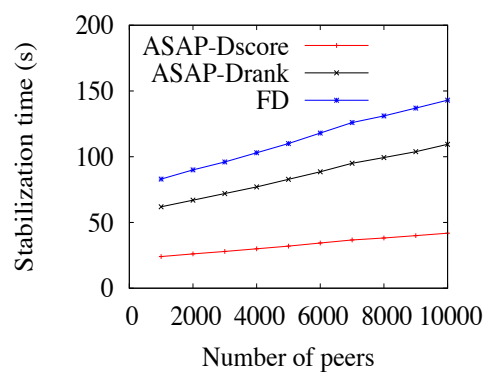

(b) Stabilization time vs. Number of peers.

(c) Total number of results vs. Number of peers.
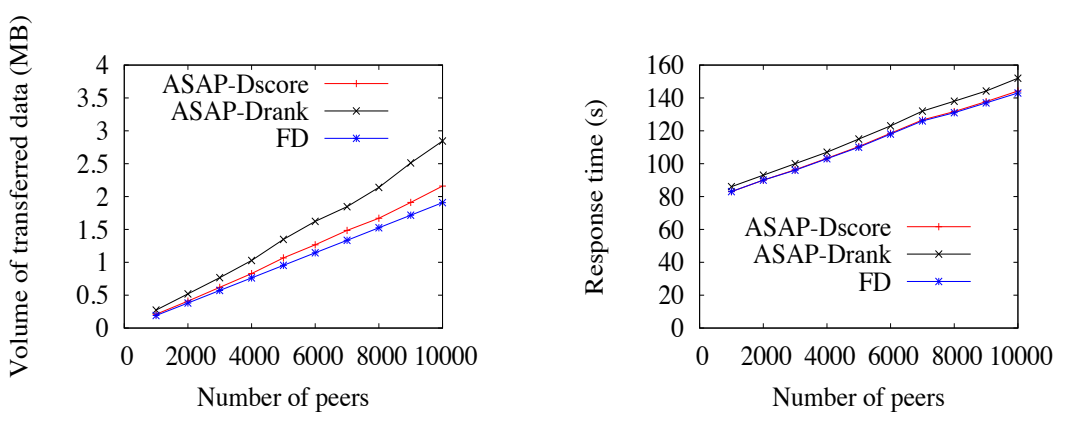

(f) Response time vs. Number of peers.

Fig. 3. Impact of number of peers on ASAP performance

time, number of answer messages, volume of transferred data, number of intermediate results and response time increase with the addition of peers. Note that the other simulation parameters are set as in Table I.

Figure 3(a) and 3(b) show respectively how cumulative quality gap and stabilization time increase with the number of peers. The results show that the cumulative quality gap of ASAPDscore and ASAP-Drank is always much smaller than that of FD, which means that ASAP returns quickly high quality results. The results also show that the stabilization time of ASAPDscore is always much smaller that of ASAP-Drank and that of FD. The reason is that ASAP-Dscore is score sensitive, so the final top- $k$ results are obtained quickly.

Figure 3(c) shows that the total number of results received by the user increases with the number of peers in the case of ASAPDscore and ASAP-Drank while it is still constant in the case of FD. This is due to the fact that FD does not provide intermediate results to users. The results also show that the number of results received by the user in case of ASAP-Dscore is smaller than that of ASAP-Drank. The main reason is that ASAP-Dscore is score sensitive in contrast to ASAP-Drank.

Figure 3(d) and Figure 3(e) show that the number of answer messages and volume of transferred data increase with the number of peers. The results show that the number of answer messages and volume of transferred data of ASAP-Drank are always higher than those of ASAP-Dscore and FD. The results also show that the differences between ASAP-Dscore and FD's number of answer messages and volume of transferred data are not significant. The main reason is that ASAP-Dscore is score sensitive in contrast to ASAP-Drank. Thus, only high quality results are bubbled up quickly.

Figure 3(f) shows how response time increases with increasing numbers of peers. The results show that the difference between ASAP-Dscore and FD response time is not significant. The results also show that the difference between ASAP-Drank and FD's response time increases slightly in favour of ASAPDrank as the number of peers increases. The reason is that ASAP-Drank induces more network traffic than ASAP-Dscore and FD.

2) Effect of $k$ : We study the effect of $k$, i.e. the number of results requested by the user, on the performance of ASAP. Using our simulator, we studied how cumulative quality gap, stabilization time and volume of transferred data evolve while increasing $k$ from 20 to 100 , with the other simulation parameters set as in Table I. The results (see Figure 4(a), Figure 4(b)) show that $k$ has very slight impact on cumulative quality gap and stabilization time of ASAP-Dscore and ASAP-Drank. The results (see Figure 4(c)) also show that by increasing $k$, the volume of transferred data of ASAP-Dscore and ASAP-Drank increase less than that of FD. This is due to the fact that ASAPDscore and ASAP-Drank prune more intermediate results when $k$ increases.

3) Data replication: We study the effect of the number of replicas, which we replicate for each data, on the performance of ASAP. Using our simulator, we studied how cumulative quality gap and stabilization time evolve while increasing the 


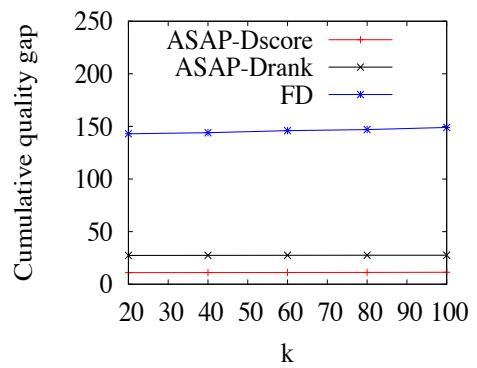

(a) Surface vs. $k$.

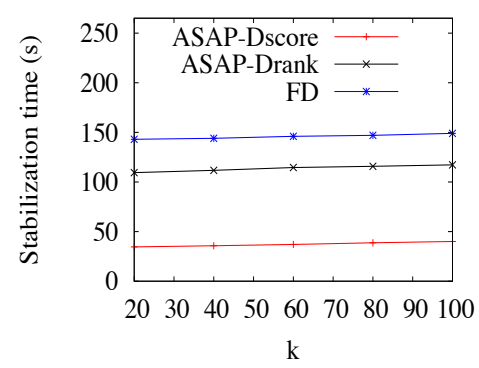

(b) Stabilization time vs. $k$.

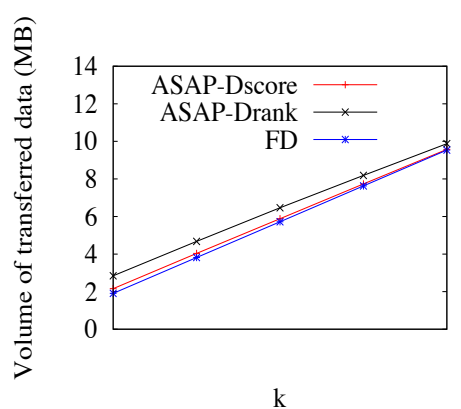

(c) Volume of transferred data vs. $k$.

Fig. 4. Impact of $k$ on ASAP performance

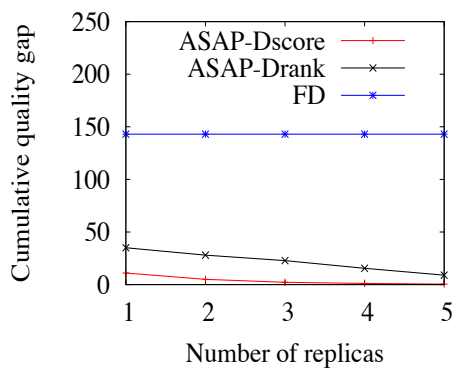

(a) Surface vs. Number of replicas.

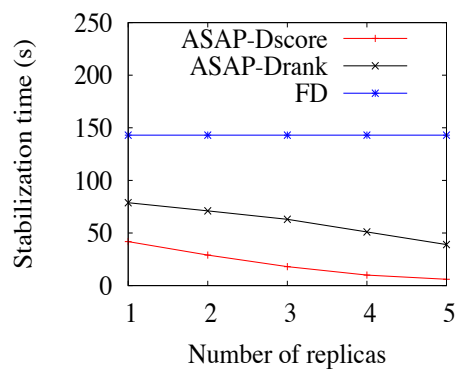

(b) Stabilization time vs. Number of replicas.

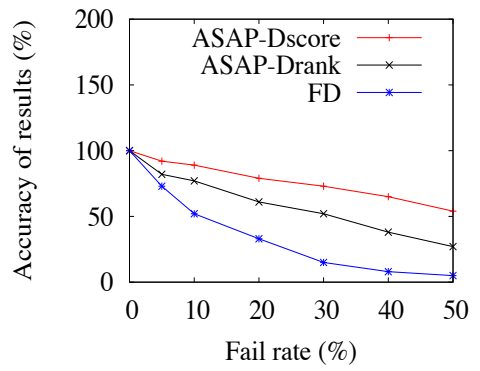

Fig. 6. Accuracy of results vs. fail rate

Fig. 5. Impact of data replication on ASAP performance

number of replicas, with the other simulation parameters set as in Table I. The results (see Figure 5(a) and Figure 5(b)) show that increasing the number of replicas for ASAP and FD decrease ASAP-Dscore and ASAP-Drank's cumulative quality gap and stabilization time. However, FD's cumulative quality gap and stabilization time are still constant. The reason is that ASAP returns quickly the results having high quality in contrast to FD which returns results only at the end of query execution. Thus, if we increase the number of replicas, ASAP finds quickly the results having high scores.

4) Effect of peers failures: In this section, we investigate the effect of peers failures on the accuracy of top- $k$ results of ASAP. In our tests, we vary the value of fail rate and investigate its effect on the accuracy of top- $k$ results. Figure 6 shows accuracy of top- $k$ results for ASAP-Dscore, ASAP-Drank and FD while increasing the fail rate, with the other parameters set as in Table I. Peers' failures have less impact on ASAP-Dscore and ASAP-Drank than FD. The reason is that ASAP-Dscore and ASAP-Drank return the high-score results to the user as soon as possible. However, when increasing the fail rate in FD, the accuracy of top- $k$ results decreases significantly because some score-lists are lost. Indeed, in FD, each peer waits for results of its children so in the case of a peer failure, all the score-lists received so far by that peer are lost.

\section{RELATED WORK}

Efficient processing of top- $k$ queries is both an important and hard problem that is still receiving much attention. Several papers have dealt with top- $k$ query processing in centralized database management systems [6], [7], [17]. In distributed systems [18], [19], [20], previous work on top- $k$ processing has focused on vertically distributed data over multiple sources, where each source provides a ranking over some attributes. The majority of the proposed approaches, such as recently [21], try to improve some limitations of the Threshold Algorithm (TA) [22]. Following the same concept, there exist some previous work for top- $k$ queries in $\mathrm{P} 2 \mathrm{P}$ over vertically distributed data. In [23], the authors propose algorithm called "ThreePhase Uniform Threshold" (TPUT) which aims at reducing communication cost by pruning away intelligible data items and restricting the number of round-trip messages between the query originator and other nodes. Later, TPUT was improved by KLEE [24]. KLEE uses the concept of bloom filters to reduce the data communicated over the network upon processing top- $k$ queries. It brings significant performance benefits with small penalties in result precision. However, theses approaches assume that data is vertically distributed over the nodes whereas we deal with horizontal data distribution.

For horizontally distributed data, there has been little work on P2P top- $k$ processing. In [5], the authors present FD, a fully distributed approach for top- $k$ query processing in unstructured $\mathrm{P} 2 \mathrm{P}$ systems. We have briefly introduced FD in section VII-A.

PlanetP [25] is the content addressable publish/subscribe service for unstructured $\mathrm{P} 2 \mathrm{P}$ communities up to ten thousand peers. PlanetP uses a gossip protocol to replicate global compact summaries of content (term-to-peer mappings) which are shared 
by each peer. The top- $k$ processing algorithm works as follows. Given a query $q$, the query originator computes a relevance ranking (using the global compact summary) of peers with respect to $q$, contacts them one by one from top to bottom of ranking and asks them to return a set of their top-scored document names together with their scores. However, in a large P2P system, keeping up-to-date the replicated index is a major problem that hurts scalability.

In [8], the authors present an index routing based top- $k$ processing technique for super-peer networks organized in an HyperCuP topology which tries to minimize the number of transfer data. The authors use queries statistics to maintain the indexes built on super-peers. However, the performance of this technique is dependent of query distribution.

In [11], the authors present SPEERTO, a framework that supports top- $k$ query processing in super-peer networks based on the use of the skyline operator. In SPEERTO, for a maximum of $K$, denoting an upper bound on the number of results requested by any top- $k$ query $(k \leq K)$, each peer computes its $K$-skyband as a pre-processing step. Each super peer maintains and aggregates the $K$-skyband sets of its peers to answer any incoming top- $k$ query. The main drawback of this approach is that each join or leave of peer may induce the recomputing of all super-peers $K$-skyband. Although these techniques are very good for super-peers systems, it cannot apply efficiently for unstructured P2P systems, since there may be no peer with higher reliability and computing power.

Zhao et al. [10] use a result caching techniques to prune network paths and answer queries without contacting all peers. The performance of this technique depends on the query distribution. They assume acyclic networks, which is restrictive for unstructured P2P systems.

\section{CONCLUSION}

This paper is the first attempt to deal with as-soon-as-possible top- $k$ query processing in $\mathrm{P} 2 \mathrm{P}$ systems. We proposed a formal definition for as-soon-as-possible top- $k$ query processing by introducing two novels notions: stabilization time and cumulative quality gap. We presented ASAP, a family of algorithms which uses a threshold-based scheme that considers the score and the rank of intermediate results to return quickly the high quality results to users. We validated ASAP through implementation and extensive experimentation. The results show that ASAP significantly outperforms baseline algorithms by returning final top- $k$ result to users in much better times. Finally, the results demonstrate that in the presence of peers' failures, ASAP provides approximative top- $k$ results with good accuracy, unlike baseline algorithms.

\section{REFERENCES}

[1] S. Androutsellis-Theotokis and D. Spinellis, "A survey of peer-to-peer content distribution technologies," ACM Computing Surveys, vol. 36, no. 4, pp. 335-371, 2004.

[2] D. Tsoumakos and N. Roussopoulos, "Analysis and comparison of p2p search methods," in Proceedings of Int. Conf. on Scalable Information Systems (Infoscale), 2006, p. 25.

[3] L. Ramaswamy, J. Chen, and P. Parate, "Coquos: Lightweight support for continuous queries in unstructured overlays," in Proceedings of IEEE International Parallel and Distributed Processing Symposium (IPDPS), 2007, pp. 1-10.
[4] S. Schmid and R. Wattenhofer, "Structuring unstructured peer-to-peer networks," in Proceedings of IEEE Int. Conf. on High Performance Computing (HiPC), 2007, pp. 432-442.

[5] R. Akbarinia, E. Pacitti, and P. Valduriez, "Reducing network traffic in unstructured p2p systems using top-k queries," Distributed and Parallel Databases, vol. 19, no. 2-3, pp. 67-86, 2006.

[6] S. Chaudhuri and L. Gravano, "Evaluating top-k selection queries," in Proceedings of Int. Conf. on Very Large Databases (VLDB), 1999, pp. 397-410.

[7] V. Hristidis, N. Koudas, and Y. Papakonstantinou, "Prefer: a system for the efficient execution of multi-parametric ranked queries," in Proceedings of ACM. Int Conf. on Management of Data (SIGMOD), 2001, pp. 259-270.

[8] W.-T. Balke, W. Nejdl, W. Siberski, and U. Thaden, "Progressive distributed top k retrieval in peer-to-peer networks," in Proceedings of Int. Conf. on Data Engineering (ICDE), 2005, pp. 174-185.

[9] K. Hose, M. Karnstedt, K.-U. Sattler, and D. Zinn, "Processing top-n queries in $\mathrm{p} 2 \mathrm{p}$-based web integration systems with probabilistic guarantees," in Proceedings of International Workshop on web and databases (WebDB), 2005, pp. 109-114.

[10] K. Zhao, Y. Tao, and S. Zhou, "Efficient top-k processing in large-scaled distributed environments," Data and Knowledge Engineering, vol. 63, no. 2, pp. 315-335, 2007.

[11] A. Vlachou, C. Doulkeridis, K. Nørvåg, and M. Vazirgiannis, "On efficient top-k query processing in highly distributed environments," in Proceedings of ACM. Int Conf. on Management of Data (SIGMOD), 2008, pp. 753-764.

[12] I. Tatarinov, Z. G. Ives, J. Madhavan, A. Y. Halevy, D. Suciu, N. N. Dalvi, X. Dong, Y. Kadiyska, G. Miklau, and P. Mork, "The piazza peer data management project," SIGMOD Record, vol. 32, no. 3, pp. 47-52, 2003.

[13] B. C. Ooi, Y. Shu, and K.-L. Tan, "Relational data sharing in peer-based data management systems," SIGMOD Record, vol. 32, no. 3, pp. 59-64, 2003.

[14] R. Akbarinia, V. Martins, E. Pacitti, and P. Valduriez, Global Data Management, 1st ed. IOS Press, 2006, ch. Design and Implementation of Atlas P2P Architecture.

[15] M. Jelasity, A. Montresor, G. P. Jesi, and S. Voulgaris, "The Peersim simulator," http://peersim.sf.net.

[16] P. K. Gummadi, S. Saroiu, and S. D. Gribble, "A measurement study of napster and gnutella as examples of peer-to-peer file sharing systems," Computer Communication Review, vol. 32, no. 1, p. 82, 2002.

[17] M. Shmueli-Scheuer, C. Li, Y. Mass, H. Roitman, R. Schenkel, and G. Weikum, "Best-effort top-k query processing under budgetary constraints," in ICDE, 2009, pp. 928-939.

[18] S. Chaudhuri, L. Gravano, and A. Marian, "Optimizing top-k selection queries over multimedia repositories," IEEE Transactions on Knowledge Data Engineering, vol. 16, no. 8, pp. 992-1009, 2004.

[19] U. Güntzer, W.-T. Balke, and W. Kießling, "Optimizing multi-feature queries for image databases," in Proceedings of Int. Conf. on Very Large DataBases (VLDB), 2000, pp. 419-428.

[20] N. Bruno, L. Gravano, and A. Marian, "Evaluating top-k queries over webaccessible databases," in Proceedings of Int. Conf on Data Engineering (ICDE), 2002, pp. 369-380.

[21] R. Akbarinia, E. Pacitti, and P. Valduriez, "Best position algorithms for top-k queries," in Proceedings of Int. Conf. on Very Large Data Bases (VLDB), 2007, pp. 495-506.

[22] R. Fagin, A. Lotem, and M. Naor, "Optimal aggregation algorithms for middleware," in Proceedings of Symposium on Principles of Database Systems (PODS), 2001, pp. 102-113.

[23] P. Cao and Z. Wan, "Efficient top-k query calculation in distributed networks," in Proceedings of Annual ACM Symposium on Principles of Distributed Computing (PODC), 2004, pp. 206-215.

[24] S. Michel, P. Triantafillou, and G. Weikum, "Klee: A framework for distributed top-k query algorithms," in Proceedings of Int. Conf. on Very Large Data Bases (VLDB), 2005, pp. 637-648.

[25] F. M. Cuenca-Acuna, C. Peery, R. P. Martin, and T. D. Nguyen, "Planetp: Using gossiping to build content addressable peer-to-peer information sharing communities," in Proceedings of IEEE Int. Symp. on HighPerformance Distributed Computing (HPDC), 2003, pp. 236-249. 